\title{
The Role of Neoadjuvant Chemotherapy in the Treatment of Nasopharyngeal Carcinoma: A Multi-institutional Retrospective Study (KROG 11-06) Using Propensity Score Matching Analysis
}

\author{
Jin Ho Song, MD ${ }^{1}$ \\ Hong-Gyun Wu, MD, PhD² \\ Bhum Suk Keam, MD ${ }^{3}$ \\ Jeong Hun Hah, $\mathrm{MD}^{4}$ \\ Yong Chan Ahn, MD, PhD \\ Dongryul Oh, MD \\ Jae Myoung Noh, MD \\ Hyo Jung Park, MD \\ Chang Geol Lee, $\mathrm{MD}^{6}$ \\ Ki Chang Keum, $\mathrm{MD}^{6}$ \\ Jihye Cha, $\mathrm{MD}^{6}$ \\ Kwan Ho Cho, MD \\ Sung Ho Moon, MD7 \\ Ji-Yoon Kim, MD ${ }^{8}$ \\ Woong-Ki Chung, MD9 \\ Young Taek Oh, MD10 \\ Won Taek Kim, MD ${ }^{11}$ \\ Moon-June Cho, MD ${ }^{12}$ \\ Chul Seung Kay, MD ${ }^{13}$ \\ Yeon-Sil Kim, MD ${ }^{14}$
}

*A list author's affiliations appears at the end of the paper.

\section{Purpose}

We compared the treatment results and toxicity in nasopharyngeal carcinoma (NPC) patients treated with concurrent chemotherapy (CCRT) alone (the CRT arm) or neoadjuvant chemotherapy followed by CCRT (the NCT arm).

\section{Materials and Methods}

A multi-institutional retrospective study was conducted to review NPC patterns of care and treatment outcome. Data of 568 NPC patients treated by CCRT alone or by neoadjuvant chemotherapy followed by CCRT were collected from 15 institutions. Patients in both treatment arms were matched using the propensity score matching method, and the clinical outcomes were analyzed.

\section{Results}

After matching, 300 patients (150 patients in each group) were selected for analysis. Higher 5-year locoregional failure-free survival was observed in the CRT arm (85\% vs. $72 \%$, $p=0.014)$. No significant differences in distant failure-free survival (DFFS), disease-free survival (DFS), and overall survival were observed between groups. In subgroup analysis, the NCT arm showed superior DFFS and DFS in stage IV patients younger than 60 years. No significant difference in compliance and toxicity was observed between groups, except the radiation therapy duration was slightly shorter in the CRT arm (50.0 days vs. 53.9 days, $\mathrm{p}=0.018$ ).

\section{Conclusion}

This study did not show the superiority of NCT followed by CCRT over CCRT alone. Because NCT could increase the risk of locoregional recurrences, it can only be considered in selected young patients with advanced stage IV disease. The role of NCT remains to be defined and should not be viewed as the standard of care.
Correspondence: Yeon Sil Kim, MD Department of Radiation Oncology, Seoul St. Mary's Hospital,

College of Medicine, The Catholic

University of Korea, 222 Banpo-daero, Seocho-gu, Seoul 06591, Korea

Tel: 82-2-2258-1501

Fax: 82-2-2258-1532

E-mail: yeonkim7@catholic.ac.kr

Received July 20, 2015

Accepted November 27, 2015

Published Online December 28, 2015
Key words

Nasopharyngeal neoplasms, Chemoradiotherapy, Induction chemotherapy, Radiotherapy, Republic of Korea 


\section{Introduction}

Nasopharyngeal carcinoma (NPC) is a relatively rare disease in South Korea, and accounts for 7.2\% to 23.1\% of head and neck cancers $[1,2]$. According to the Korean National Cancer Registry Report in 2011, its incidence in South Korea was 0.8 per 100,000 [3], lower than in endemic areas, such as southern China, southeast Asia, and north Africa, but higher than that of Western countries [2,4]. NPC in endemic areas differs from Western NPC in that more than $90 \%$ of patients have non-keratinizing and undifferentiated carcinoma, which is more frequently related to Epstein-Barr virus (EBV) [1]. Although South Korea is a non-endemic area, some clinicopathologic characteristics of NPC are shared with NPC in endemic areas, including strong relation with EBV and a lower prevalence of keratinizing carcinoma than in Western countries [1,2].

NPC is usually considered unresectable because of its anatomical location, thus radiation therapy (RT) plays a major role in its treatment $[5,6]$. The current standard treatment strategy for locally advanced NPC is concurrent chemoradiotherapy (CCRT) with or without adjuvant chemotherapy, which was determined based on the superior results of randomized trials comparing RT alone and CCRT [7-9]. However, even when treated with CCRT, 3-year progression-free survival (PFS) is still only around 70\% [7,8]. Recent advances in RT technology, such as intensity-modulated radiation therapy (IMRT), make it possible to increase the radiation dose with the same or lower toxicity and achieve higher local control rates $[5,10]$. In addition, because of the high rate of distant metastasis in NPC patients, there is a resurgence of interest in adjuvant and neoadjuvant chemotherapy (NCT) for advanced NPC in combination with CCRT. However, because of the diverse chemotherapy regimens, schedules, and mixed results reported, it is difficult to determine whether adjuvant chemotherapy or NCT can improve treatment outcome in NPC [5,11-16].

In this study, we compared the treatment results and toxicities of CCRT alone (CRT arm) and NCT followed by CCRT (NCT arm) in primary NPC patients.

\section{Materials and Methods}

\section{Patients}

To review the NPC patterns of care and treatment outcomes in South Korea, a multi-institutional retrospective study (Korean Radiation Oncology Group [KROG] 11-06) was conducted through the Head and Neck Committee of Korean Society of Radiation Oncology (KOSRO). Data of 1474 primary NPC patients treated at 15 institutions in South Korea between September 1988 and October 2011 were collected. Clinical and pathological data were collected from medical records. All patients satisfied the following criteria: (1) biopsy-proven NPC; (2) stage I to IVb according to the American Joint Committee on Cancer (AJCC) cancer staging, 6th edition; (3) age over 18 years; and (4) Eastern Cooperative Oncology Group system (ECOG) performance status (PS) $\leq 2$. Patients who had undergone previous surgery, radiotherapy to the head and neck, and those with distant metastasis before treatment initiation were excluded.

Prior to patient enrollment, institutional review board approval was obtained at each participating center and data were transferred to the KROG Data Center (National Cancer Center, Goyang, Korea). Radiotherapy, chemotherapy, toxicity, recurrence, and survival reports of all patients were reviewed according to a centralized quality-assurance program.

For this study, we selected 583 patients who satisfied the following inclusion criteria: (1) stage II to IVb by the AJCC cancer staging, 6th edition; (2) treated by CCRT alone or NCT followed by CCRT; and (3) non-receipt of any type of adjuvant chemotherapy after CCRT. Of these 583 patients, 15 patients with insufficient data for analysis of treatment outcome were excluded. The remaining 568 patients were finally included in the analysis, 380 patients were included in the CRT arm and 188 patients in the NCT arm.

\section{Patient selection using the propensity score matching method}

Because parts of the baseline characteristics between the CRT and NCT arm were statistically different, including histologic type, hospital size, and RT techniques (Table 1), the propensity score matching (PSM) method was used to control the imbalance. Nine covariates were entered in the propensity model, including age, sex, hospital size, ECOG PS, histologic type, T stage, N stage, RT technique, and the CCRT regimen. Propensity scores were calculated using a non-parsimonious multivariable logistic regression model to estimate the conditional probability. Then a 1:1 match between the CRT and NCT arm was performed using the nearest available neighbor matching. After PSM, 150 pairs of patients were matched successfully, and 300 patients were included for analysis.

\section{Study endpoints and statistical analysis}

We analyzed the patients and treatment characteristics, compliances, toxicities, and treatment outcomes. Histologic 
Table 1. Baseline patient characteristics in the pre- and post-propensity score matching cohort

\begin{tabular}{|c|c|c|c|c|c|c|}
\hline \multirow{2}{*}{ Characteristic } & \multicolumn{3}{|c|}{ Before propensity score matching } & \multicolumn{3}{|c|}{ After propensity score matching } \\
\hline & CRT $(n=380)$ & NCT $(n=188)$ & p-value & CRT (n=150) & $\mathrm{NCT}(\mathrm{n}=150)$ & p-value \\
\hline \multicolumn{7}{|l|}{ Sex } \\
\hline Male & $266(70.0)$ & $144(76.6)$ & 0.099 & $111(74.0)$ & $111(74.0)$ & $>0.999$ \\
\hline Female & $114(30.0)$ & $44(23.4)$ & & $39(26.0)$ & $39(26.0)$ & \\
\hline \multicolumn{7}{|l|}{ Age } \\
\hline Median (range, yr) & $52(19-86)$ & $50(20-73)$ & 0.223 & $51.5(19-77)$ & $50(19-72)$ & 0.648 \\
\hline Mean \pm SD & $51.4 \pm 13.1$ & $50.0 \pm 12.1$ & & $50.8 \pm 13.2$ & $50.5 \pm 11.6$ & \\
\hline \multicolumn{7}{|l|}{ ECOG PS } \\
\hline 0 & $65(17.1)$ & $34(18.1)$ & 0.539 & $27(18.0)$ & $25(16.7)$ & 0.963 \\
\hline 1 & $303(79.7)$ & $151(80.3)$ & & $119(79.3)$ & $122(81.3)$ & \\
\hline 2 & $12(3.2)$ & $3(1.6)$ & & $4(2.7)$ & $3(2.0)$ & \\
\hline \multicolumn{7}{|l|}{ WHO histology } \\
\hline I & $77(20.3)$ & $17(9.0)$ & $<0.001$ & $15(10.0)$ & 17 (11.3) & 0.502 \\
\hline IIa & $108(28.4)$ & $75(39.9)$ & & $59(39.3)$ & $58(38.7)$ & \\
\hline $\mathrm{IIb}$ & $187(49.2)$ & $84(44.7)$ & & $70(46.7)$ & $71(47.3)$ & \\
\hline Not classified & $8(2.1)$ & $12(6.4)$ & & $6(4.0)$ & $4(2.7)$ & \\
\hline \multicolumn{7}{|l|}{ T stage } \\
\hline 1 & $95(25.0)$ & $54(28.7)$ & 0.308 & $38(25.3)$ & $40(26.7)$ & 0.389 \\
\hline 2 & $102(26.8)$ & $39(20.7)$ & & $34(22.7)$ & $34(22.7)$ & \\
\hline 3 & $86(22.6)$ & 39 (20.7) & & $36(24.0)$ & $35(23.3)$ & \\
\hline 4 & $97(25.5)$ & $56(29.8)$ & & $42(28.0)$ & $41(27.3)$ & \\
\hline \multicolumn{7}{|l|}{ N stage } \\
\hline 0 & 44 (11.6) & $16(8.5)$ & 0.366 & $15(10.0)$ & $13(8.7)$ & 0.410 \\
\hline 1 & $104(27.4)$ & $50(26.6)$ & & $42(28.0)$ & $41(27.3)$ & \\
\hline 2 & $172(45.3)$ & $98(52.1)$ & & $71(47.3)$ & $75(50.0)$ & \\
\hline 3 & $60(15.8)$ & $24(12.8)$ & & $22(14.7)$ & $21(14.0)$ & \\
\hline \multicolumn{7}{|l|}{ AJCC stage } \\
\hline II & $38(10.0)$ & $13(6.9)$ & 0.480 & $14(9.3)$ & $12(8.0)$ & 0.914 \\
\hline III & $194(51.1)$ & $99(52.7)$ & & 77 (51.3) & $79(52.7)$ & \\
\hline IV & $148(38.9)$ & $76(40.4)$ & & $59(39.3)$ & $59(39.3)$ & \\
\hline \multicolumn{7}{|l|}{ Hospital size } \\
\hline Large & $264(69.5)$ & $104(55.3)$ & 0.001 & $97(64.7)$ & $93(62.0)$ & 0.637 \\
\hline Small & $116(30.5)$ & $84(44.7)$ & & $53(35.3)$ & $57(38.0)$ & \\
\hline \multicolumn{7}{|l|}{ RT method } \\
\hline Non-IMRT & $231(60.8)$ & $63(33.5)$ & $<0.001$ & $52(34.7)$ & $57(38.0)$ & 0.456 \\
\hline IMRT & 149 (39.2) & $125(66.5)$ & & $98(65.3)$ & $93(62.0)$ & \\
\hline \multicolumn{7}{|l|}{ CRT regimen } \\
\hline Cisplatin alone & $322(84.7)$ & 159 (84.6) & 0.883 & $131(87.3)$ & $126(84.0)$ & 0.411 \\
\hline Combined & $58(15.3)$ & 29 (15.4) & & $19(12.7)$ & $24(16.0)$ & \\
\hline \multicolumn{7}{|l|}{ NCT regimen } \\
\hline FP & - & $36(19.1)$ & $\mathrm{N} / \mathrm{A}$ & - & $32(21.3)$ & $\mathrm{N} / \mathrm{A}$ \\
\hline F or P+taxane & - & $64(34.0)$ & & - & $61(40.7)$ & \\
\hline $\mathrm{FP}+$ taxane & - & 78 (41.4) & & - & $48(32.0)$ & \\
\hline Other & - & $10(5.3)$ & & - & $9(6.0)$ & \\
\hline
\end{tabular}

Values are presented as number (\%) unless otherwise indicated. CRT, concurrent chemoradiotherapy arm; NCT, neoadjuvant chemotherapy arm; SD, standard deviation; ECOG PS, Eastern Cooperative Oncology Group performance status; WHO, World Health Organization; AJCC, American Joint Committee on Cancer; RT, radiation therapy; IMRT, intensity-modulated radiotherapy; F, 5-fluorouracil; $\mathrm{P}$, cisplatin; N/A, not assessed. 
type was defined according to the World Health Organization (WHO) classification 2005 [17]. Tumor responses were recorded according to WHO criteria [18]. Acute and late treatment toxicities were assessed using the National Cancer Institute's Common Terminology Criteria for Adverse Events (CTCAE) ver. 3.0 [19]. Only the incidences of toxicities $\geq$ grade 3 were recorded.

Overall survival (OS) was defined as the time from pathologic diagnosis of NPC to any cause of death; all deaths from any cause were counted as events and patients who remained alive were treated as censored at the date of last follow-up. Disease-free survival (DFS) was defined as time from pathologic diagnosis to time of first failure at any site or any death. Locoregional failure-free survival (LRFFS) and distant failure-free survival (DFFS) were also analyzed, and were defined as any recurrence in the nasopharyngeal and / or cervical region or any failure at a distant site. Survival proportions were estimated using the Kaplan-Meier method.

Standardized differences were estimated for all baseline covariates before and after matching to assess pre-match imbalance and post-match balance. In the matched data, paired comparisons were performed using McNemar's test for binary variables and Wilcoxon signed rank test for continuous variables. The log-rank test and the Cox proportional hazards model were used to identify prognostic factors independently associated with survival and to estimate hazard ratios (HR). Two-sided p-values of $<0.05$ were considered statistically significant. Statistical analyses were performed using the software package SPSS ver. 21.0 (SPSS Inc., Chicago, IL) or R software ver. 2.15.3 (R Foundation for Statistical Computing, Vienna, Austria; http://www.r-project. org).

\section{Results}

\section{Patients and treatment characteristics}

A summary of patient and treatment characteristics before and after PSM is shown in Table 1. Of 300 patients selected after PSM, there were 222 male patients $(74.0 \%)$ with a median age of 51 years (range, 19 to 77 years). The ECOG PS were $0-1$ in 293 patients $(97.6 \%$ ), and 2 in only seven patients (2.3\%). WHO type I (keratinizing carcinoma) tumors were found in 32 patients $(10.7 \%)$, and were evenly distributed after PSM. The AJCC tumor stage was II in 26 patients (8.7\%), III in 156 patients (52.0\%), and IV in 118 patients (39.3\%).

More patients in the NCT arm were treated with IMRT at small hospitals before PSM of which "small" means the average number of daily treated RT patients is under 100 . After PSM, patients were evenly distributed, and 191 patients $(63.7 \%)$ were treated with IMRT. The most commonly used chemotherapy regimen during the CCRT was cisplatin alone for 257 patients $(85.7 \%)$. During NCT, taxane combined with cisplatin or 5-fluorouracil (5-FU), or both was most frequently used in 109 patients (62.7\%). The other 41 patients $(27.3 \%)$ received a non-taxane containing regimen during NCT.

\section{Response and survival}

Tumor response data were collected only after completion of CCRT, and not after completion of NCT. It was evaluated in 290 patients $(96.7 \%)$ after median 1.4 months follow-up (range, 0.1 to 39.0 months), and showed similar results between groups with no statistical difference $(\mathrm{p}=0.180)$. Complete response was achieved in 87 patients $(60.0 \%)$ in the CRT arm, and 92 patients (63.4\%) in the NCT arm. Objective response (complete or partial response) was achieved in 140 patients (96.6\%) in the CRT arm, and 142 patients (97.9\%) in the NCT arm. Progressive disease was found in two patients $(1.4 \%)$, and both patients were in the NCT arm and had stage IV disease.

The OS, DFS, LRFFS, and DFFS between treatment arms are shown in Fig. 1 after a median follow-up of 35.9 months (range, 6.4 to 18.2 months) in the CRT arm, and 33.8 months (range, 6.4 to 152.6 months) in the NCT arm. The 5-year LRFFS was $85 \%$ in the CRT arm and $72 \%$ in the NCT arm, which was a statistically significant difference $(\mathrm{p}=0.014)$. Compared to LRFFS, lower 5-year DFFS was observed in the CRT arm compared with the NCT arm (65\% vs. $76 \%)$; however, it was not statistically significant $(\mathrm{p}=0.390)$. No difference in OS and DFS was observed between the CRT and NCT arm. The 5-year OS and DFS were $72.1 \%, 52.2 \%$ in the CRT arm, and $81.1 \%, 57.1 \%$ in the NCT arm, respectively ( $\mathrm{p}=0.340$ and $\mathrm{p}=0.978$, respectively).

\section{Prognostic factors affecting survival}

The results of multivariate analysis of the prognostic factors on survival are shown in Table 2. The $\mathrm{T}$ and $\mathrm{N}$ stage were significant factors affecting the survival. The T stage affected not only the LRFFS but also the DFFS, DFS, and OS, while the N-stage affected the DFFS and DFS only. The difference of LRFFS between treatment arms also showed statistical significance in multivariate analysis with $\mathrm{HR}$ of 2.21 (95\% confidence interval [CI], 1.19 to 4.12; $\mathrm{p}=0.012$ ). Other factors including sex, ECOG PS, WHO histology, and RT method did not show statistical significance in multivariate analysis. 
A

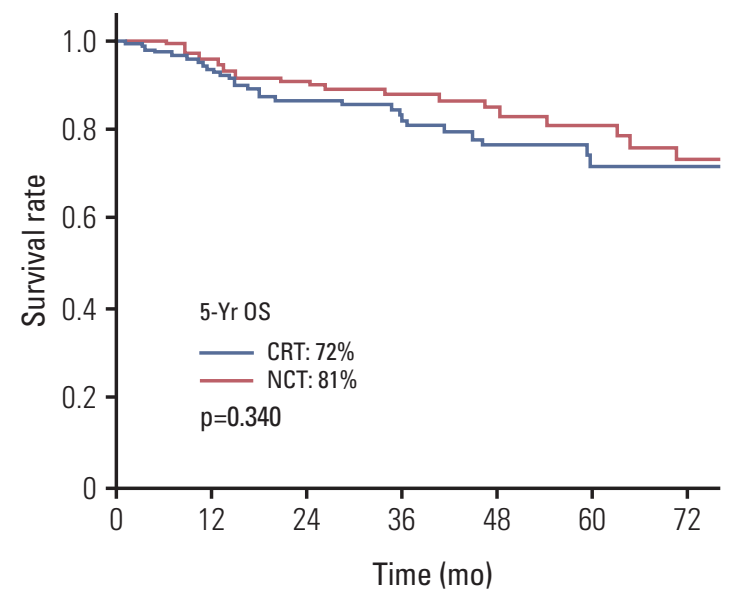

No. at risk

$\begin{array}{llllllll}\text { CRT } & 150 & 124 & 98 & 68 & 51 & 31 & 18\end{array}$

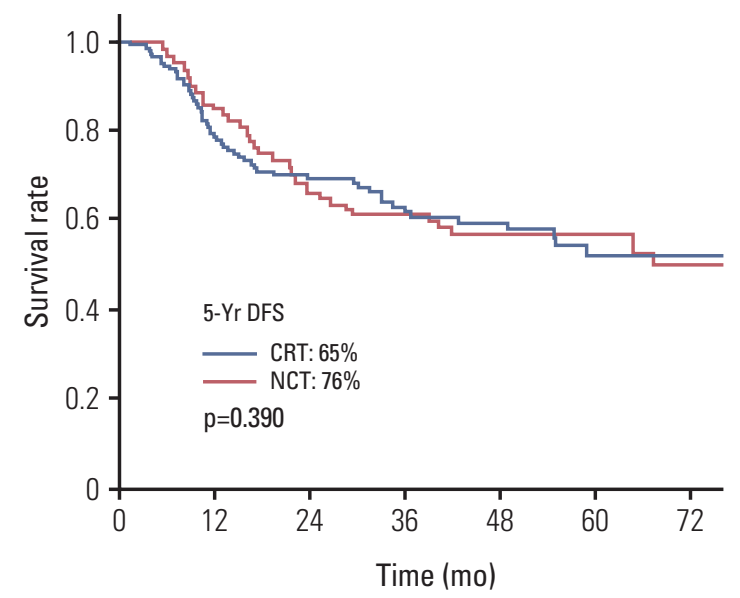

No. at risk

$\begin{array}{llllllll}\text { CRT } & 150 & 105 & 81 & 53 & 40 & 22 & 14 \\ \text { NCT } & 150 & 124 & 79 & 49 & 33 & 27 & 20\end{array}$

C

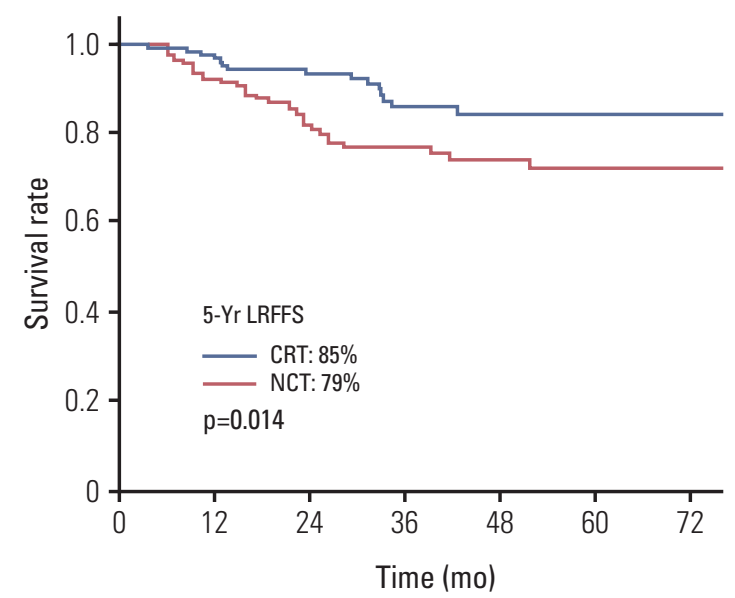

No. at risk

$\begin{array}{llllllll}\text { CRT } & 150 & 121 & 94 & 61 & 46 & 28 & 16\end{array}$

B 
Table 2. Multivariate analysis of prognostic factors in the propensity-matched cohort

\begin{tabular}{|c|c|c|c|c|c|c|c|c|}
\hline \multirow[t]{2}{*}{ Factor } & \multicolumn{2}{|c|}{ Overall survival } & \multicolumn{2}{|c|}{ Disease-free survival } & \multicolumn{2}{|c|}{$\begin{array}{l}\text { Locoregional } \\
\text { failure-free survival }\end{array}$} & \multicolumn{2}{|c|}{$\begin{array}{c}\text { Distant } \\
\text { failure-free survival }\end{array}$} \\
\hline & Adjust HR & p-value & Adjust HR & p-value & Adjust HR & p-value & Adjust HR & p-value \\
\hline Treatment arm (CRT vs. NCT) & $0.74(0.53-1.30)$ & 0.297 & $1.02(0.70-1.47)$ & 0.939 & $2.21(1.19-4.12)$ & 0.012 & $0.84(0.52-1.38)$ & 0.844 \\
\hline Sex (male vs. female) & $1.46(0.7-2.75)$ & 0.246 & $0.90(0.58-1.41)$ & 0.648 & $1.25(0.65-2.42)$ & 0.503 & $0.61(0.31-1.18)$ & 0.140 \\
\hline Age ( $<60$ yr vs. $\geq 60$ yr $)$ & $1.02(1.00-1.05)$ & 0.087 & $1.01(0.99-1.03)$ & 0.258 & $1.03(1.00-1.05)$ & 0.069 & $1.00(0.98-1.02)$ & 0.841 \\
\hline ECOG PS (0-1 vs. 2) & $0.63(0.08-4.91)$ & 0.662 & $1.26(0.45-3.55)$ & 0.662 & $2.13(0.48-9.48)$ & 0.319 & $0.92(0.22-3.90)$ & 0.913 \\
\hline WHO histology (I vs. II) & $1.32(0.55-3.19)$ & 0.535 & $1.21(0.68-2.15)$ & 0.528 & $1.10(0.45-2.67)$ & 0.830 & $1.81(0.76-4.30)$ & 0.180 \\
\hline T stage (1-2 vs. 3-4) & $2.54(1.38-4.70)$ & 0.003 & $2.28(1.53-3.39)$ & $<0.001$ & $2.16(1.16-4.01)$ & 0.015 & $2.11(1.26-3.53)$ & 0.005 \\
\hline N stage (0-1 vs. $2-3)$ & $1.56(0.85-2.89)$ & 0.153 & $2.00(1.30-3.08)$ & 0.002 & $1.47(0.76-2.82)$ & 0.252 & $2.30(1.29-4.10)$ & 0.005 \\
\hline RT method (non-IMRT vs. IMRT) & $0.93(0.52-1.69)$ & 0.819 & $1.00(0.67-1.49)$ & 0.996 & $0.99(0.53-1.85)$ & 0.970 & $0.94(0.56-1.58)$ & 0.821 \\
\hline
\end{tabular}

HR, hazard ratio; CRT, concurrent chemoradiotherapy arm; NCT, neoadjuvant chemotherapy arm; ECOG PS, Eastern Cooperative Oncology Group performance status; WHO, World Health Organization; RT, radiation therapy; IMRT, intensity-modulated radiotherapy.

Table 3. Subgroup analysis by prognostic factors in the propensity-matched cohort

\begin{tabular}{|c|c|c|c|c|c|c|c|c|}
\hline \multirow[t]{2}{*}{ Factor (CRT vs. NCT arm) } & \multicolumn{2}{|c|}{ Overall survival } & \multicolumn{2}{|c|}{ Disease-free survival } & \multicolumn{2}{|c|}{$\begin{array}{l}\text { Locoregional } \\
\text { failure-free survival }\end{array}$} & \multicolumn{2}{|c|}{$\begin{array}{c}\text { Distant } \\
\text { failure-free survival }\end{array}$} \\
\hline & Adjust HR & p-value & Adjust HR & p-value & Adjust HR & p-value & Adjust HR & p-value \\
\hline \multicolumn{9}{|l|}{ Age } \\
\hline$<60$ yr (116 vs. 110$)$ & $0.78(0.38-1.58)$ & 0.486 & $0.79(0.50-1.24)$ & 0.302 & $1.48(0.69-3.19)$ & 0.314 & $0.64(0.36-1.15)$ & 0.138 \\
\hline$\geq 60$ yr (44 vs. 40$)$ & $0.80(0.32-2.02)$ & 0.804 & $1.65(0.85-3.21)$ & 0.138 & $4.04(1.33-12.26)$ & 0.014 & $1.48(0.58-3.76)$ & 0.407 \\
\hline \multicolumn{9}{|l|}{ AJCC stage } \\
\hline II-III (91 vs. 91) & $0.45(1.67-1.21)$ & 0.116 & $1.28(0.74-2.19)$ & 0.378 & $3.34(1.33-8.37)$ & 0.010 & $1.06(0.53-2.11)$ & 0.873 \\
\hline IV (59 vs. 59) & $1.08(0.53-2.16)$ & 0.840 & $0.80(0.47-1.34)$ & 0.390 & $1.49(0.62-3.57)$ & 0.377 & $0.54(0.27-1.09)$ & 0.088 \\
\hline $\begin{array}{l}\text { Age }<60 \text { yr and } \\
\text { AJCC stage IV ( } 44 \text { vs. } 46)\end{array}$ & $0.81(0.33-2.02)$ & 0.652 & $0.56(0.30-1.02)$ & 0.058 & $1.06(0.36-3.08)$ & 0.918 & $0.46(0.21-0.99)$ & 0.049 \\
\hline
\end{tabular}

CRT, concurrent chemoradiotherapy arm; NCT, neoadjuvant chemotherapy arm; HR, hazard ratio; AJCC, American Joint Committee on Cancer.

AJCC stage IV disease, the adjusted HR for DFFS was 0.46 (95\% CI, 0.21 to $0.99 ; p=0.049$ ). The DFS also favored NCT arms in these patients with HR of 0.56 (95\% CI, 0.30 to 1.02) with marginal significance ( $\mathrm{p}=0.058$ ). However, the OS was not different. The survival outcomes of these patients are shown in Fig. 2.

\section{Compliance and toxicity}

Because only patients who had completed their RT schedule were selected after PSM, the compliance was analyzed on the whole patient cohort and is shown in Table 4. Of the 568 patients, 15 patients $(2.6 \%)$ did not receive the planned RT dose and treatment was interrupted. Seven of these 15 patients were in the CRT arm, and eight were in the NCT arm. A higher percentage was observed in the NCT arm $(4.3 \%$ vs. $1.8 \%)$, but without statistical significance $(\mathrm{p}=0.091)$. The reasons for RT interruption were poor general condition (9 patients), severe mucositis (2 patients), patient refusal (2 patients), and renal failure (1 patient). Durations of RT were similar in the two arms with an average of 53.5 days in the CRT arm and 52.8 days in the NCT arm for the entire cohort $(\mathrm{p}=0.621)$. However, in the PSM cohort, the average RT duration was 3.9 days longer in the NCT arm (50.0 vs. 53.9 days, $\mathrm{p}=0.018$ ).

The chemotherapy schedule during CCRT was interrupted in 94 patients $(16.5 \%), 61$ patients in the CRT arm and 33 patients in the NCT arm. Chemotherapy tolerance during 


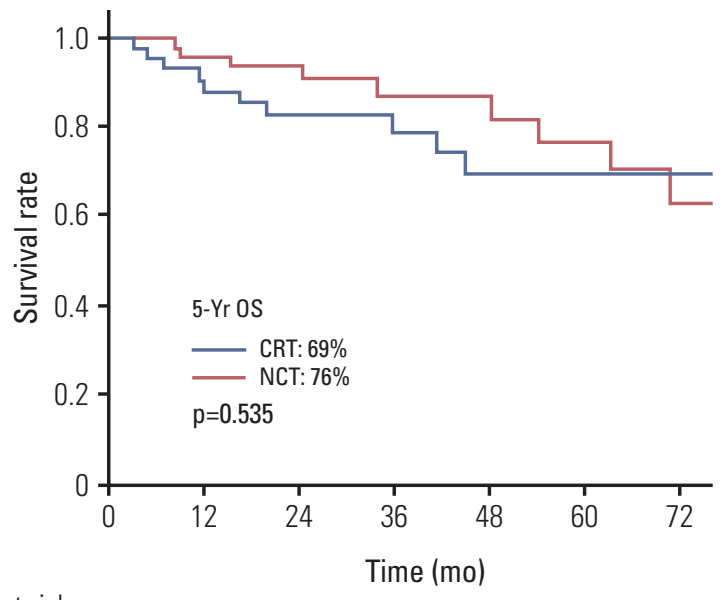

No. at risk

$\begin{array}{llllllll}\text { CRT } & 44 & 37 & 28 & 20 & 14 & 8 & 6\end{array}$

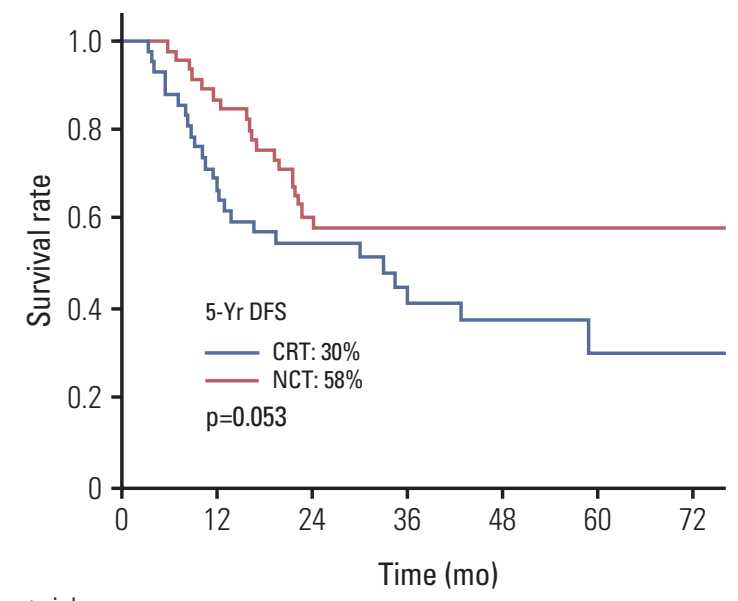

No. at risk

$\begin{array}{rrrrrrrr}\text { CRT } & 44 & 27 & 21 & 12 & 9 & 4 & 4 \\ \text { NCT } & 46 & 40 & 23 & 14 & 11 & 9 & 7\end{array}$

\section{C}

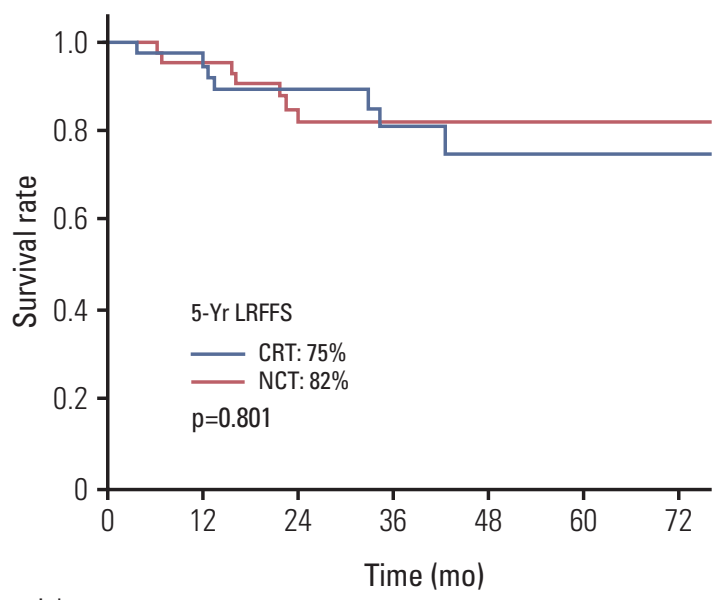

No. at risk

$\begin{array}{rrrrrrrr}\text { CRT } & 44 & 36 & 27 & 17 & 12 & 7 & 5 \\ \text { NCT } & 46 & 43 & 28 & 18 & 15 & 13 & 9\end{array}$

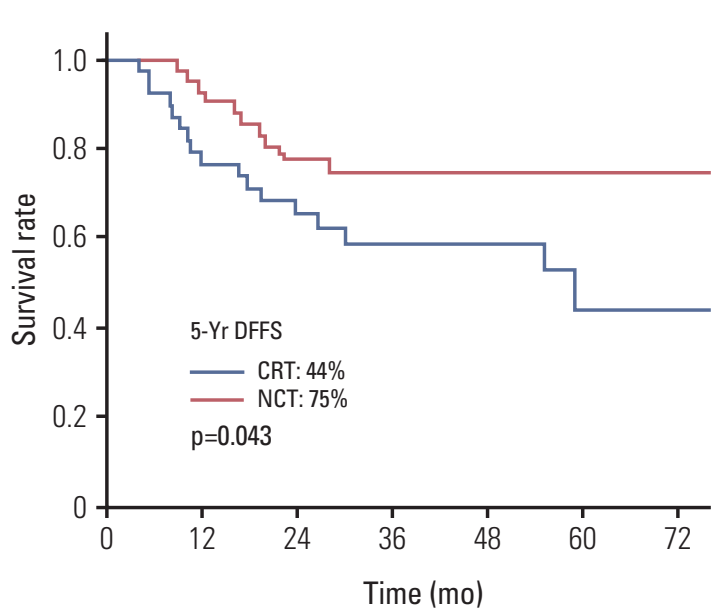

No. at risk

$\begin{array}{rrrrrrrr}\text { CRT } & 44 & 29 & 22 & 15 & 11 & 5 & 5 \\ \text { NCT } & 46 & 41 & 28 & 17 & 13 & 9 & 7\end{array}$

Fig. 2. Overall survival (OS) (A), disease-free survival (DFS) (B), locoregional failure-free survival (LRFFS) (C), and distant failure-free survival (DFFS) (D) curves for the concurrent chemoradiotherapy arm (CRT) and neoadjuvant chemotherapy arm (NCT) arms in subgroup with stage IV disease patients younger than 60 years.

CCRT was similar in the two arms for any chemotherapy regimen (Table 4). Five patients $(2.7 \%)$ received only one cycle of the planned NCT course because of no response or progression in four patients and nephrotoxicity in one.

Acute and late toxicities are shown in Table 5. Toxicity was analyzed in the PSM cohort to balance the use of IMRT and the difference of chemotherapy regimen. The most frequent $\geq$ grade 3 acute toxicities were neutropenia and mucositis. Incidences of $\geq$ grade 3 acute toxicity showed no difference between the treatment arms. However, higher incidence of $\geq$ grade 4 hematologic toxicity was observed in the NCT arm $(3.3 \%$ vs. $0.7 \%)$. The most frequent late toxicity was xerostomia, followed by soft tissue fibrosis, radiation necrosis, and ototoxicity. However, the most fatal toxicities were radiation 
Table 4. Radiotherapy and chemotherapy compliance in the entire cohort

\begin{tabular}{|c|c|c|c|}
\hline Type of treatment & CRT $(n=380)$ & NCT $(n=188)$ & p-value \\
\hline Radiation interruption & $7(1.8)$ & $8(4.3)$ & 0.091 \\
\hline \multicolumn{4}{|c|}{ Duration, mean (range, day) } \\
\hline Entire cohort & $53.5(17-165)$ & $52.8(13-158)$ & 0.621 \\
\hline PSM cohort & $50.0(31-98)$ & $53.9(34-117)$ & 0.018 \\
\hline \multicolumn{4}{|c|}{ Chemotherapy during CRT } \\
\hline Total interruption & $61(16.1)$ & $33(17.6)$ & 0.651 \\
\hline \multicolumn{4}{|l|}{ Cisplatin weekly } \\
\hline $1-4$ cycles & $24(18.8)$ & $23(21.9)$ & 0.550 \\
\hline$\geq 5$ cycles & $104(81.2)$ & $82(78.1)$ & \\
\hline \multicolumn{4}{|l|}{ Cisplatin, every 3 wk } \\
\hline 1 cycle & $17(8.9)$ & $3(5.7)$ & 0.447 \\
\hline$\geq 2$ cycles & $174(91.1)$ & $50(94.3)$ & \\
\hline \multicolumn{4}{|c|}{ Platinum +5 -FU or taxane } \\
\hline $1-2$ cycles & $15(40.5)$ & $5(35.7)$ & 0.752 \\
\hline$\geq 3$ cycles & $22(59.5)$ & $9(64.3)$ & \\
\hline \multicolumn{4}{|c|}{ Chemotherapy during NCT } \\
\hline 1 cycle & - & $5(2.7)$ & - \\
\hline 2 cycles & - & $35(22.9)$ & \\
\hline$\geq 3$ cycles & - & $148(77.1)$ & \\
\hline
\end{tabular}

Values are presented as number (\%) unless otherwise indicated. CRT, concurrent chemoradiotherapy arm; NCT, neoadjuvant chemotherapy arm; PSM, propensity score matching; 5-FU, 5-fluorouracil.

Table 5. Grade 3 or higher acute and late toxicity in the propensity-matched cohort

\begin{tabular}{lccc} 
Type of toxicity & CRT $(\mathbf{n}=\mathbf{1 5 0})$ & NCT (n=150) & p-value \\
Acute toxicity (grade 3/4) & & & \\
$\quad$ Hematologic & & & 0.857 \\
$\quad$ Grade 3 & $17(11.3)$ & $18(12.0)$ & 0.214 \\
$\quad$ Grade 4 & $1(0.7)$ & $5(3.3)$ & 0.448 \\
Anorexia/Nausea/Vomiting & $5(3.3)$ & $2(1.3)$ & 0.767 \\
Mucositis/Odynophagia & $27(18.0)$ & $29(19.3)$ & 0.750 \\
Dermatitis & $4(2.7)$ & $6(4.0)$ & $>0.999$ \\
Xerostomia & $4(2.7)$ & $4(2.7)$ & 0.813 \\
Any acute toxicity & $57(38.0)$ & $59(39.3)$ & 0.723 \\
Late toxicity (grade 3/4) & & & $>0.999$ \\
Soft tissue fibrosis & $5(3.3)$ & $3(2.0)$ & 0.777 \\
Radionecrosis/Neuropathy & $2(1.3)$ & $1(0.7)$ & $>0.999$ \\
Xerostomia & $6(4.0)$ & $7(4.7)$ & 0.840 \\
Ototoxicity & $1(0.7)$ & $2(1.3)$ & \\
Any late toxicity & $14(9.3)$ & $13(8.7)$ & 0.498 \\
Grade 5 toxicity & & & $>0.999$ \\
Radionecrosis/Neuropathy & 0 & $2(1.3)$ & $>0.999$ \\
Neutropenia/Infection & $1(0.7)$ & 0 & $>0.999$ \\
\hline Not reported & $2(1.3)$ & $2(1.3)$ & $4(2.7)$ \\
\hline Any grade 5 toxicity & $3(2.0)$ & & \\
\hline
\end{tabular}

Values are presented as number (\%). Treatment toxicities were assessed using the National Cancer Institute's Common Terminology Criteria for Adverse Events (ver. 3.0). CRT, concurrent chemoradiotherapy arm; NCT, neoadjuvant chemotherapy arm. 
necrosis and neutropenia. Overall, treatment-related death occurred in seven patients (2.3\%). No difference in late toxicity was observed between treatment arms.

\section{Discussion}

To improve the treatment outcomes of NPC, the addition of adjuvant chemotherapy or NCT to CCRT has been widely studied [5]. NCT is believed to be superior to adjuvant chemotherapy in some theoretical aspects: (1) compliance with the entire course of chemotherapy is higher for NCT; (2) penetration of chemotherapeutic agents into tumor tissues might be limited due to the disruption of native blood vessels after RT; and (3) NCT might kill subclinical micrometastases before definitive local treatment $[5,20]$. For these reasons, NCT has been an attractive strategy for many researchers.

Before the Intergroup 0099 study, which showed superior results for CCRT as compared with RT alone [7], several studies compared NCT followed by RT and RT alone. Although a reduction in relapse-free and disease-specific survival was observed with NCT in some studies, no differences in OS or treatment failure pattern were observed [21,22].

After CCRT became the standard treatment for advanced NPC, there were questions with regard to whether addition of NCT to CCRT could improve outcomes, and, as a result, several phase II trials were conducted. Hui et al. [11] reported on a comparative randomized phase II study on NCT with docetaxel and cisplatin (TP) followed by CCRT versus CCRT alone. Three-year DFS was not significantly different $(88.2 \%$ in the NCT arm and 59.5\% in the CRT arm, $\mathrm{p}=0.12$ ). However, 3-year OS was statistically superior in the NCT arm ( $94.1 \%$ vs. $67.7 \%)$.

The Hellenic Cooperative Oncology Group (HeCOG) also reported the results of a comparative phase II study on NCT with a cisplatin/epirubicin/paclitaxel regimen followed by CCRT versus CCRT alone [14]. In analysis of the data of 141 patients, no difference in terms of compliance, response, 3-year DFS and OS was observed between the two arms.

A meta-analysis on 11 studies conducted by Liang et al. [15], which included 1096 NPC patients, compared the results of NCT followed by CCRT versus CCRT with or without adjuvant chemotherapy. This study also showed that NCT followed by CCRT did not significantly improve OS, LRFFS, or DFFS.

In the current study, we also failed to demonstrate a better outcome for NCT versus CCRT alone. Although the DFFS, DFS, and OS did not differ between groups, lower LRFFS was observed in the NCT arm. This result seems to reflect the theoretical disadvantages of NCT. The disadvantages of NCT include the selection of resistant tumor cell clones, triggering accelerated repopulation, and reduced compliance and increased toxicity during CCRT.

In our study, even though the toxicities were similar between the two arms, RT was interrupted more frequently in the NCT arm (4.3\%) than in the CRT arm (1.8\%), and the overall treatment time of RT was also longer in the NCT arm in the PSM cohort. In our study, in comparison of the local control rate according to RT duration, significantly lower LRFFS was observed in patients with RT duration longer than 50 days $(82.8 \%$ vs. $73.3 \%, p=0.034)$. The advantage of NCT by reducing the distant failure was only observed in stage IV disease patients and those younger than 60 years. This result of subgroup analysis was consistent with that of the study reported by Zhang et al. [23]. They also conducted a PMS study of NPC patients in China, which showed improved DFFS only in T4N1-2 or N3 patients $(86.1 \%$ vs. $74.9 \%, \mathrm{p}=0.034$ ), favoring NCT.

However, there are some limitations of our study that warrant consideration. First, the heterogeneous NCT regimen could diminish the positive effect of NCT. Non-taxane containing regimen was also used in $27.3 \%$ of patients, which was unfavorable if we consider the recent impressive outcomes by addition of taxane to the NCT regimens. In the EORTC 24971/TAX 323 study, triple combinations of docetaxel, cisplatin, and 5-FU (TPF) showed superior PFS and OS rates compared with cisplatin and 5-FU in other head and neck cancers [24]. Based on these findings, Ekenel et al. [13] used TP for the NCT regimen in NPC patients, and reported a 3-year OS of $94.9 \%$ and a PFS of $84.7 \%$. Bae et al. [12], who also reported the results of NCT using the TPF regimen followed by CCRT in Korea, achieved 3-year OS and PFS rates of $86.1 \%$ and $75.6 \%$, respectively [12], which were slightly lower than results obtained in endemic areas, but were nevertheless remarkable.

The second limitation of our study was the relatively imperfect data on toxicity. This was due to the retrospective nature of data collection from several institutions, and it resulted in comparative low toxicity rates than other literatures. However, similar toxicity rates between two treatment arms have also been reported in other studies. The HeCOG group study reported no significant differences in acute toxicities after CCRT [14], even though thrombocytopenia was more common in the NCT arm and dermatitis was more common in the CRT arm. However, the rates of all other toxicities were similar in both arms. In a meta-analysis conducted by Liang et al. [15], the toxicity results showed that the risk ratio of grade 3-4 neutropenia was $1.90(95 \% \mathrm{CI}, 1.24$ to 2.92) in favor of the CRT arm rather than the NCT arm. However, other toxicities were similar. 


\section{Conclusion}

In conclusion, this study did not show the superiority of NCT followed by CCRT over CCRT alone. Because NCT could increase the risk of locoregional recurrences, it can only be considered in selected young patients with advanced stage IV disease. The role of NCT in addition to CCRT remains to be defined and is currently not the standard of care. The results of phase III trials are needed in order to draw a firm conclusion.

\section{Conflicts of Interest}

Conflict of interest relevant to this article was not reported.

\section{Acknowledgments}

The statistical analysis was supported by the Catholic Research Coordinating Center of the Korea Health 21 R\&D Project (A070001), Ministry of Health \& Welfare, Republic of Korea.

\section{Author Details}

${ }^{1}$ Department of Radiation Oncology, Gyeongsang National University Hospital and Gyeongsang National University School of Medicine, Jinju, Departments of ${ }^{2}$ Radiation Oncology, ${ }^{3}$ Internal Medicine, and ${ }^{4}$ Otolaryngology-Head and Neck Surgery, Seoul National University Hospital, Cancer Research Institute, Seoul National University College of Medicine, Seoul, '5epartment of Radiation Oncology, Samsung Medical Center, Sungkyunkwan University School of Medicine, Seoul, ${ }^{6}$ Department of Radiation Oncology, Yonsei University College of Medicine, Seoul, ${ }^{7}$ Department of Radiation Oncology, Research Institute and Hospital, National Cancer Center, Seoul, ${ }^{8}$ Department of Radiation Oncology, Yeouido St. Mary's Hospital, College of Medicine, The Catholic University of Korea, Seoul, ${ }^{9}$ Department of Radiation Oncology, Chonnam National University Medical School, Gwangju, ${ }^{10}$ Department of Radiation Oncology, Ajou University School of Medicine, Suwon, ${ }^{11}$ Department of Radiation Oncology, Pusan National University School of Medicine, Busan, ${ }^{12}$ Department of Radiation Oncology, Chungnam National University School of Medicine, Daejeon, ${ }^{13}$ Department of Radiation Oncology, Incheon St. Mary's Hospital, College of Medicine, The Catholic University of Korea, Incheon, ${ }^{14}$ Department of Radiation Oncology, Seoul St. Mary's Hospital, College of Medicine, The Catholic University of Korea, Seoul, Korea

\section{References}

1. Jeon YK, Lee BY, Kim JE, Lee SS, Kim CW. Molecular characterization of Epstein-Barr virus and oncoprotein expression in nasopharyngeal carcinoma in Korea. Head Neck. 2004;26: 573-83.

2. Kim YJ, Go H, Wu HG, Jeon YK, Park SW, Lee SH. Immunohistochemical study identifying prognostic biomolecular markers in nasopharyngeal carcinoma treated by radiotherapy. Head Neck. 2011;33:1458-66.

3. The Korea Central Cancer Registry, National Cancer Center. Annual report of cancer statistics in Korea in 2011. Gwacheon: Ministry of Health and Welfare; 2012.

4. Lee NK, Park YJ, Yang DS, Yoon WS, Lee S, Kim CY. Longterm results of 2-dimensional radiation therapy in patients with nasopharyngeal cancer. J Korean Soc Ther Radiol Oncol. 2010;28:193-204.

5. Spratt DE, Lee N. Current and emerging treatment options for nasopharyngeal carcinoma. Onco Targets Ther. 2012;5: 297-308.

6. Kim YS, Kim BS, Jung SL, Lee YS, Kim MS, Sun DI, et al. Radiation therapy combined with (or without) cisplatin-based chemotherapy for patients with nasopharyngeal cancer: 15years experience of a single institution in Korea. Cancer Res Treat. 2008;40:155-63.

7. Al-Sarraf M, LeBlanc M, Giri PG, Fu KK, Cooper J, Vuong T, et al. Chemoradiotherapy versus radiotherapy in patients with advanced nasopharyngeal cancer: phase III randomized Intergroup study 0099. J Clin Oncol. 1998;16:1310-7.

8. Lin JC, Jan JS, Hsu CY, Liang WM, Jiang RS, Wang WY. Phase III study of concurrent chemoradiotherapy versus radiotherapy alone for advanced nasopharyngeal carcinoma: positive effect on overall and progression-free survival. J Clin Oncol. 2003;21:631-7.

9. Chan AT, Leung SF, Ngan RK, Teo PM, Lau WH, Kwan WH, et al. Overall survival after concurrent cisplatin-radiotherapy compared with radiotherapy alone in locoregionally advanced nasopharyngeal carcinoma. J Natl Cancer Inst. 2005;97:536-9.

10. Kong M, Hong SE, Choi J, Kim Y. Comparison of survival rates between patients treated with conventional radiotherapy and helical tomotherapy for head and neck cancer. Radiat Oncol J. 2013;31:1-11. 
11. Hui EP, Ma BB, Leung SF, King AD, Mo F, Kam MK, et al. Randomized phase II trial of concurrent cisplatin-radiotherapy with or without neoadjuvant docetaxel and cisplatin in advanced nasopharyngeal carcinoma. J Clin Oncol. 2009;27: 242-9.

12. Bae WK, Hwang JE, Shim HJ, Cho SH, Lee JK, Lim SC, et al. Phase II study of docetaxel, cisplatin, and 5-FU induction chemotherapy followed by chemoradiotherapy in locoregionally advanced nasopharyngeal cancer. Cancer Chemother Pharmacol. 2010;65:589-95.

13. Ekenel M, Keskin S, Basaran M, Ozdemir C, Meral R, Altun $\mathrm{M}$, et al. Induction chemotherapy with docetaxel and cisplatin is highly effective for locally advanced nasopharyngeal carcinoma. Oral Oncol. 2011;47:660-4.

14. Fountzilas G, Ciuleanu E, Bobos M, Kalogera-Fountzila A, Eleftheraki AG, Karayannopoulou G, et al. Induction chemotherapy followed by concomitant radiotherapy and weekly cisplatin versus the same concomitant chemoradiotherapy in patients with nasopharyngeal carcinoma: a randomized phase II study conducted by the Hellenic Cooperative Oncology Group (HeCOG) with biomarker evaluation. Ann Oncol. 2012; 23:427-35.

15. Liang ZG, Zhu XD, Tan AH, Jiang YM, Qu S, Su F, et al. Induction chemotherapy followed by concurrent chemoradiotherapy versus concurrent chemoradiotherapy with or with-out adjuvant chemotherapy for locoregionally advanced nasopharyngeal carcinoma: meta-analysis of 1,096 patients from 11 randomized controlled trials. Asian Pac J Cancer Prev. 2013;14: 515-21.

16. Wee CW, Keam B, Heo DS, Sung MW, Won TB, Wu HG. Locoregionally advanced nasopharyngeal carcinoma treated with intensity-modulated radiotherapy plus concurrent weekly cisplatin with or without neoadjuvant chemotherapy.
Radiat Oncol J. 2015;33:98-108.

17. Thompson LD. Update on nasopharyngeal carcinoma. Head Neck Pathol. 2007;1:81-6.

18. Miller AB, Hoogstraten B, Staquet M, Winkler A. Reporting results of cancer treatment. Cancer. 1981;47:207-14.

19. Trotti A, Colevas AD, Setser A, Rusch V, Jaques D, Budach V, et al. CTCAE v3.0: development of a comprehensive grading system for the adverse effects of cancer treatment. Semin Radiat Oncol. 2003;13:176-81.

20. Kong L, Hu C, Niu X, Zhang Y, Guo Y, Tham IW, et al. Neoadjuvant chemotherapy followed by concurrent chemoradiation for locoregionally advanced nasopharyngeal carcinoma: interim results from 2 prospective phase 2 clinical trials. Cancer. 2013;119:4111-8.

21. Ma J, Mai HQ, Hong MH, Min HQ, Mao ZD, Cui NJ, et al. Results of a prospective randomized trial comparing neoadjuvant chemotherapy plus radiotherapy with radiotherapy alone in patients with locoregionally advanced nasopharyngeal carcinoma. J Clin Oncol. 2001;19:1350-7.

22. Hareyama M, Sakata K, Shirato H, Nishioka T, Nishio M, Suzuki K, et al. A prospective, randomized trial comparing neoadjuvant chemotherapy with radiotherapy alone in patients with advanced nasopharyngeal carcinoma. Cancer. 2002;94:2217-23.

23. Zhang LN, Gao YH, Lan XW, Tang J, OuYang PY, Xie FY. Effect of taxanes-based induction chemotherapy in locoregionally advanced nasopharyngeal carcinoma: A large scale propensity-matched study. Oral Oncol. 2015;51:950-6.

24. Vermorken JB, Remenar E, van Herpen C, Gorlia T, Mesia R, Degardin $\mathrm{M}$, et al. Cisplatin, fluorouracil, and docetaxel in unresectable head and neck cancer. N Engl J Med. 2007;357: 1695-704. 